\title{
Immediate Effects of Stretching for Iliopsoas Muscles on Lum- bopelvic-Hip Kinematics during Gait: A Randomised Controlled Trial Using Subjects with Non-Specific Low Back Pain
}

\author{
Koya Mine ${ }^{1,2 *}$, Keio Ishiguro ${ }^{1}$ and Takashi Nakayama ${ }^{1,2}$ \\ ${ }^{1}$ Department of Physical Therapy, Tokyo University of Technology, Japan \\ ${ }^{2}$ International Centre for Allied Health Evidence, University of South Australia, Australia
}

*Corresponding author: Koya Mine, Department of Physical Therapy, School of Health Sciences, Tokyo University of Technology, 5-23-22 Nishi-kamata, Ota Ward, Tokyo, 144-8535, Tokyo, Japan, E-mail: mineky@stt.teu.ac.jp

\begin{abstract}
Background: Stretching for iliopsoas muscles is commonly performed for patients with non-specific low back pain, which tends to be provoked by extension-related movements. However, it is unclear whether stretching can immediately alter lumbopelvic-hip kinematics of patients during gait.

Aim: To investigate immediate effects of active static stretching for iliopsoas muscles on lumbopelvic-hip kinematics of patients with non-specific low back pain.

Methods: This study was a randomised controlled trial in a laboratory setting. Ten male young patients (age $20.6 \pm 0.7$, body mass index $22.8 \pm 2.4$ ) with non-specific low back pain provoked by lumbar extension related movements participated in this study. All participants had reduced bilateral hip extension according to modified Thomas test. Active static stretching was performed for one out of the two limbs $(20$ $\mathrm{s}$, three times). The other sides served as a control group. A six-camera Vicon motion-analysis system was used to measure peak ranges of hip extension, pelvic anterior tilt and rotation, and lumbar extension and rotation during gait.

Results: Both two groups did not show any significant change in lumbopelvic-hip kinematics after stretching or control interventions. Between-group comparisons showed no significant difference in any outcome measures in terms of $p$ values. However, our additional analysis using effect sizes implied a significantly greater increase in peak lumbar rotation angle in the stretching group compared to the control group.

Conclusions: This study suggests that a single set of active static stretching might not immediately improve hip extension or reduce lumbopelvic movements of patients with non-specific low back pain during gait. Further study with better methodological rigour is required to test the inconclusive findings in this study.
\end{abstract}

\section{Keywords}

Non-specific low back pain, Lumbopelvic-hip kinematics, Gait, Static stretching, Iliopsoas muscles

\section{Introduction}

Low back pain is one of the most common and burdensome musculoskeletal conditions in the modern world $[1,2]$. The majority of low back pain is called non-specific low back pain, where pathophysiological causes of symptoms are hard to be specified through medical investigations [3]. In other words, peripheral nociceptive sources of pain cannot be definitively identified by the use of modern imaging techniques. However, various attempts have been made to classify non-specific low back pain according to characteristic postures and movement patterns of patients by many authors [4-7]. These authors commonly report a potential existence of patients whose pain tends to be provoked by lumbar extension, as a subcategory of non-specific low back pain.

The existence of such a subcategory of non-specific low back pain is a commonly held belief among many health professionals dealing with neuro musculoskeletal disorders [4-7]. Patients in this subcategory are typically described to assume excessive lumbar extension and pelvic anterior tilt during static and dynamic movement tasks. It has been hypothesised that this type of posture and movement pattern may be contributed by weak ab- 
dominal muscles and stiff hip flexors, such as iliopsoas, rectus femoris and tensor fascia lata [4-6]. On this basis, static stretching for anterior hip muscles are occasionally chosen as a treatment option to improve extensibility of these muscles and reduce excessive mechanical stress to lumbar spine during particular movements of patients with particular low back pain. For instance, normal gait and other functional movements, such as forward lunge on a flat surface requires a certain amount of hip extension [8,9]. If this movement component is restricted, it might be compensated by excessive pelvic anterior tilt, rotation and lumbar extension, potentially leading to more mechanical stress to lumbar spine. Repetitive extension and rotation stress in lumbar spine might potentially contribute to specific low back pain by spondylolysis in some young athletes [10].

One randomised controlled trial suggested that ten-week stretching program to improve hip flexor extensibility can increase hip extension range of motion during gait at a flat surface [11]. On the other hand, one randomised controlled trial showed no significant change in lumbopelvic-hip kinematic parameters during functional movement tasks after a six-week stretching intervention, despite significant increase in passive hip extension range of motion [9]. Since these studies recruited only healthy subjects without any musculoskeletal conditions, these findings cannot be directly applied to patients with non-specific low back pain. To date, there is no research evidence whether stretching for iliopsoas muscles can immediately alter lumbopelvic-hip kinematics of patients with non-specific low back pain.

The purpose of this study was to examine the immediate effects of active static stretching for iliopsoas muscle on lumbopelvic-hip kinematics of patients with non-specific low back pain during gait. We hyposthesised that stretching for iliopsoas muscles would immediately increase hip extension and reduce pelvic anterior tilt and rotation, and lumbar extension and rotation of subjects with non-specific low back pain and reduced hip extension.

\section{Methods}

\section{Research design}

This study was a single-blind randomised controlled trial with evidence level II in the hierarchy of National Health and Medical Research Council [12].

\section{Participants}

Participants were recruited from undergraduate physiotherapy students who belonged to Department of Physical Therapy, School of Health Sciences at the Tokyo University of Technology from 2016 to 2017. Inclusion criteria were as follows; male adults aged over 20 years, the presence of non-specific low back pain exacerbated by extension-related movements, such as walking, running or sustained standing, and positive modified Thomas test on both sides. Pain intensity was assessed as an average pain during the last one month, using 11-point numerical rating scale (0 to 10). Modified Thomas test was regarded as positive when hip extension range was less than 0 degree measured by a goniometer (Tsutsumi Manufacturing Company, Japan) $[4,6]$. This muscle length test has been reported to be reliable to measure hip extension range of motion [13]. Exclusion criteria was the presence of nocturnal pain, unexplained weight loss, saddle anesthesia, bowel and bladder dysfunctions, bilateral leg symptoms, marked spinal deformity, leg length discrepancy greater than $2.0 \mathrm{~cm}$, positive straight leg raise test, or inability to perform active stretching for iliopsoas stretching without pain. All screening tests were performed by the same physiotherapist, who had completed Masters degree in musculoskeletal and sports physiotherapy, in order to identify eligible subjects. After the screening assessments, ten out of 12 subjects met the inclusion criteria and agreed to participate in this study (refer to Figure 1). Two students were excluded due to negative findings in modified Thomas test.

Written consents to participation were achieved after sufficient explanation about the purpose and methods of

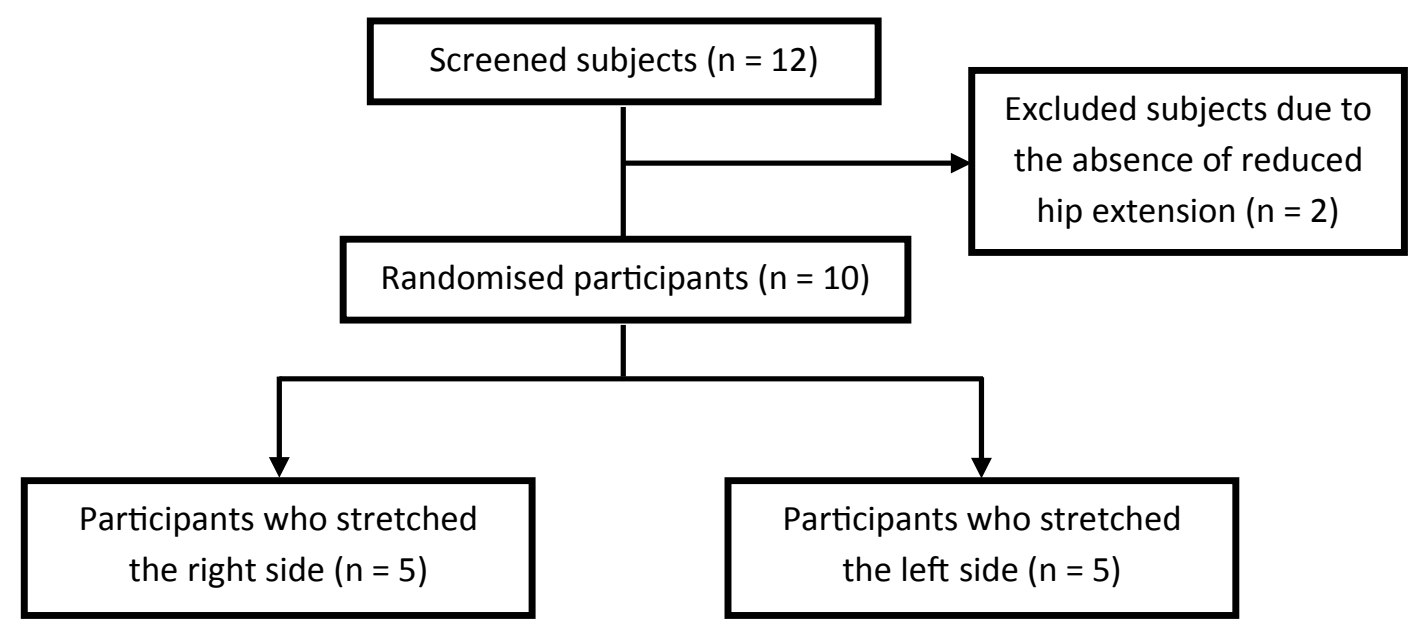

Figure 1: Flowchart of the study. 


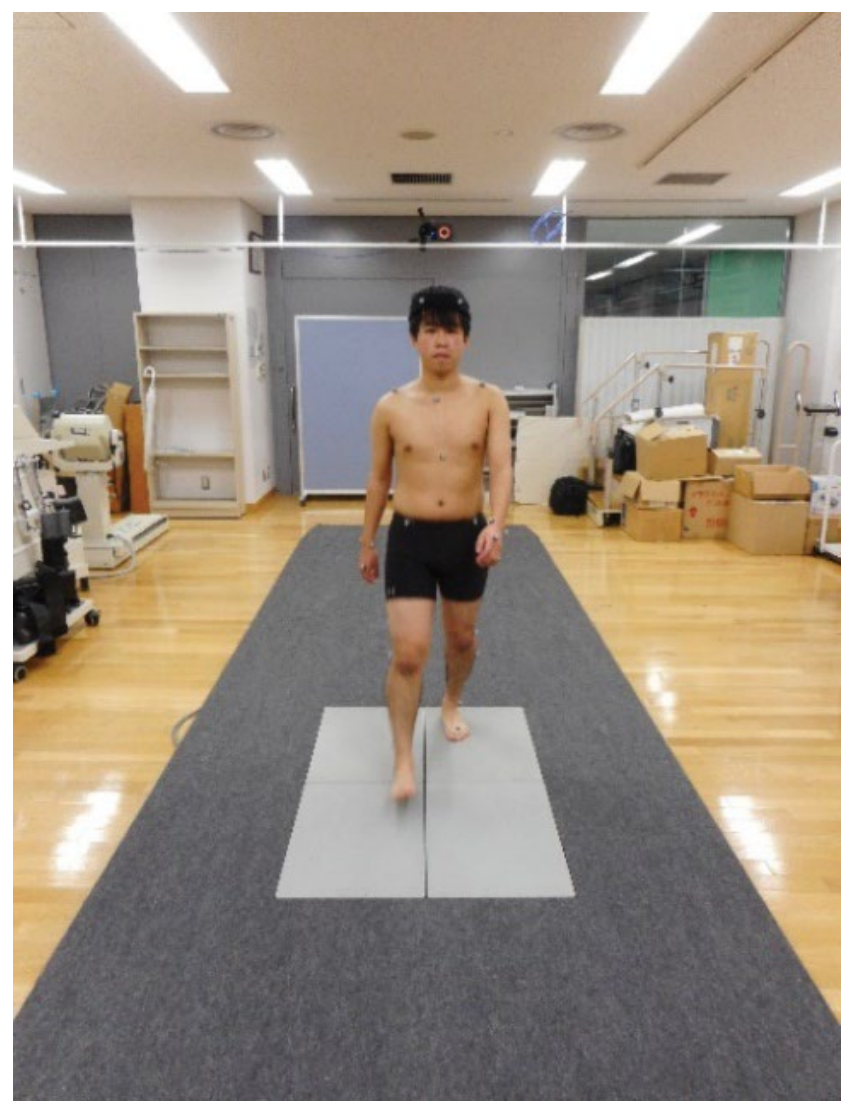

Figure 2: Assessment of lumbopelvic-hip kinematics during gait.

the study, and an assurance of privacy and confidentiality. This study was performed following an approval from an ethical committee at the Tokyo University of Technology (approval number E16HS-022).

\section{Instrumentation}

Testing sessions were carried out in the same laboratory room at Tokyo University of Technology. A six-camera Vicon motion-analysis system and Vicon Nexus software version 1.7.1 (Vicon, UK) were utilised to evaluate and process the kinematic characteristics of gait among participants. A camera frequency was set at $100 \mathrm{~Hz}$. This 3-D motion analysis is a widely used technique which has previously been shown to provide valid and reliable measurements for gait analysis [14]. In testing sessions, a total of 35 reflective markers (1.4-cm diameter) were attached at bony landmarks of the whole body, based on Plug-In-Gait model (Vicon, UK) (see Figure 2). Participants were instructed to wear only non-reflective firm-fitting elastic short pants. After all markers were positioned on the given landmarks, participants were asked to walk on an 8.1-metre laboratory walkway at their own usual speeds. Data was extracted from one gait cycle around the middle point of the walkway. The motion-analysis system was operated by the same one author in the assessments for all the participants. The raw data of marker positions were digitally filtered with a fourth order low-pass Butterworth filter with 6 $\mathrm{Hz}$ cut-off frequency. Step length, step width and speed

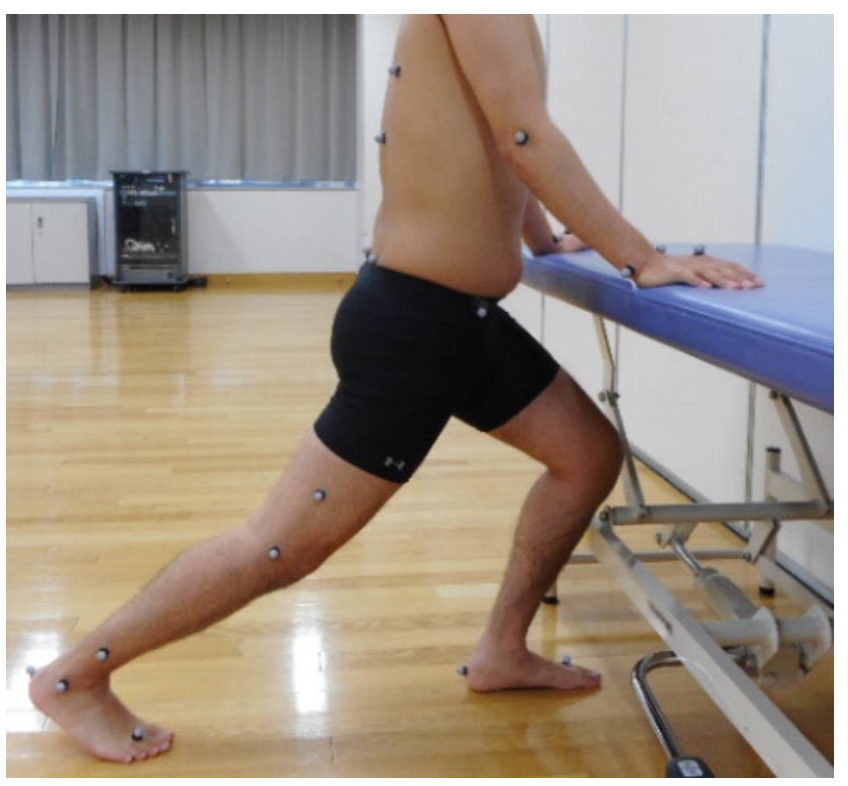

Figure 3: Stretching for iliopsoas muscle.

were calculated based on the data of the one gait cycle. Step length was calculated as the anterior-posterior distance between the most prominent parts of calcaneus at heel-contact of one foot to the most prominent part of the calcaneus of the contralateral foot at heel-contact. Step width was determined as the medial-lateral distance between the same two bony landmarks. Fourth order?, cut-off? ( $\mathrm{Hz})$, low pass filter.

\section{Procedures}

After the inclusion of eligible participants, they were randomly assigned to one of two groups by using Excel 2016 (Microsoft, USA), which enabled concealed allocation (see Figure 1). In one group, subjects performed stretching the right sides, whereas the other group stretched the left sides. Sides which were not stretched, collectively served as a control group. Primary outcome measures of interest included the following; peak angle in hip extension, pelvic anterior tilt and ipsilateral rotation, and lumbar extension and contralateral rotation. Rotation was defined as the movements in transverse plane. Anterior or posterior tilt of pelvis was defined as movements which occur in sagittal plane. Peak angle was calculated based on data, extracted from mid-stance to toe-off of the ipsilateral lower limb in one gait cycle in the middle point of a walkway. In this study, mid-stance phase was defined as $10-30 \%$ of gait cycle, initiated by toe-off of the contralateral foot [15]. Participants were instructed to walk on the 8.1-metre walkway at a comfortable speed, looking at the straight direction (Figure 2). Followed by a few practices for familiarization, gait was assessed twice, using the Vicon system (Vicon, UK). Mean values for peak joint angles were calculated using data from three trials for pre- and post-intervention data respectively. Assessments of gait were performed immediately before and after stretching interventions in the same manner. 
A stretching intervention for iliopsoas muscle was shown in Figure 3. Participants extended one hip until they felt stretching feeling in anterior hip, and maintained the posture for $20 \mathrm{~s}$ in a static way. Sides to be stretched were chosen randomly using Excel 2016 (Microsoft, USA). Subjects used a 0.75-metre table to bear their body weights on, so that they could stretch iliopsoas muscles at end range with less muscle contraction to maintain the posture. They were instructed to repeat this stretching three times, with ten-second rests in a relaxed standing position between stretching. The whole stretching intervention lasted for approximately one minute and $30 \mathrm{~s}$. When subjects were performing active stretching, the assessor was out of the testing room, which enabled blinding for assessors. Due to the nature of active stretching intervention, it was not possible to blind participants, who also acted as 'therapists' in this study [16].

\section{Statistical analyses}

The results are presented as mean \pm Standard Deviation (SD) values. To test the reproducibility of the kinematic assessments using VICON, Intraclass Coefficients (ICCS) were calculated for each outcome measure. An independent t-test was used to confirm the baseline comparability. Based on the valid assumption that parametric tests are robust enough not to require normal distribution of the data, parametric tests were consistently used to examine statistical significance [17]. A paired t-test was performed for differences between pre- and post-intervention data within each condition. An independent t-test was used to assess differences between the two conditions. A p value of less than 0.05 was used to determine statistical significance. Clinical significance of the effects was also assessed using between-group effect sizes (Hedges' g) and 95\% Confidence Intervals $(\mathrm{Cl})[18,19]$. Effect sizes were catego- rised as small $(<0.3)$, moderate $(<0.5)$ and large $(>0.8)$ [20]. All statistical analyses were conducted using SPSS version 21.0 (IBM, USA).

\section{Results}

The flowchart of the experimental process is shown in Figure 1. Characteristics of the participants with non-specific low back pain were described in Table 1. The participants were aged $20.6 \pm 0.7$, with height of $172.9 \pm 6.5 \mathrm{~cm}$, weight of $67.6 \pm 9.6$, and body mass index of $22.8 \pm 2.4$. Subjective pain intensity of the included subjects was $4.1 \pm 0.9$. Characteristics of the stretching group and the control group were shown in Table 2. As a result of randomisation, five participants were allocated to stretch their left lower limbs, whilst the other five participants stretched their right limbs. Dominant lower-limbs were right side for all participants. Mean hip extension angles in Modified Thomas test were -7.0 $\pm 3.3^{\circ}$ in the stretched limbs and $-7.5 \pm 2.5^{\circ}$ in the control limbs $(p=0.72)$. There was no statistical difference in step length between the two groups $(p=0.88)$. All subjects completed both pre- and post-measurements and there was no drop out.

The results of kinematic parameters in lumbopelvic-hip regions in the two groups are summarized in Table 3. There was no significant difference in the baseline

Table 1: Characteristics of participants with non-specific low back pain.

\begin{tabular}{|l|l|}
\hline & Participants (n = 10) \\
\hline Age & $20.6 \pm 0.7$ \\
\hline Height $(\mathrm{cm})$ & $172.0 \pm 6.5$ \\
\hline Body weight $(\mathrm{kg})$ & $67.6 \pm 9.6$ \\
\hline Pain intensity & $4.1 \pm 0.9$ \\
\hline Stride (pre-intervention) (m) & $1.4 \pm 0.1$ \\
\hline Step width (pre-intervention) (cm) & $13.0 \pm 4.7$ \\
\hline Speed (pre-intervention) (m/s) & $1.3 \pm 0.2$ \\
\hline
\end{tabular}

Table 2: Characteristics of the two groups.

\begin{tabular}{|l|l|l|}
\hline & Stretching group (Ten limbs) & Control group (Ten limbs) \\
\hline Ratio of limbs to be stretched $(\mathrm{R}: \mathrm{L})$ & $5: 5$ & $5: 5$ \\
\hline Modified Thomas test (degrees) & $-7.0 \pm 3.3$ & $-7.5 \pm 2.5$ \\
\hline Step length (pre-intervention) $(\mathrm{cm})$ & $70.0 \pm 6.9$ & $70.0 \pm 5.7$ \\
\hline
\end{tabular}

Table 3: A comparison of pre- and post-intervention data in the two groups.

\begin{tabular}{|l|l|l|l|}
\hline Stretching group & Pre-intervention $\mathbf{( n = 1 0 )}$ & Post-intervention $\mathbf{( n = 1 0 )}$ & $\mathbf{p ~ v a l u e ~}$ \\
\hline Hip extension peak angle & $13.65 \pm 8.23^{\circ}$ & $13.92 \pm 8.86^{\circ}$ & 0.53 \\
\hline Pelvic anterior tilt peak angle & $9.37 \pm 4.47^{\circ}$ & $9.31 \pm 4.39^{\circ}$ & 0.89 \\
\hline Pelvic rotation peak angle & $6.53 \pm 2.54^{\circ}$ & $6.78 \pm 2.71^{\circ}$ & 0.58 \\
\hline Lumbar extension peak angle & $10.36 \pm 5.96^{\circ}$ & $10.16 \pm 5.40^{\circ}$ & 0.74 \\
\hline Lumbar rotation peak angle & $7.33 \pm 2.20^{\circ}$ & $8.11 \pm 2.19^{\circ}$ & 0.26 \\
\hline Step length (cm) & $70.0 \pm 6.9$ & $70.5 \pm 5.2$ & 0.35 \\
\hline Control group & Pre-intervention $(\mathbf{n}=10)$ & Post-intervention $(\mathbf{n}=10)$ & $\mathbf{p ~ v a l u e ~}$ \\
\hline Hip extension peak angle & $13.01 \pm 5.01^{\circ}$ & $13.07 \pm 4.88^{\circ}$ & 0.85 \\
\hline Pelvic anterior tilt peak angle & $9.39 \pm 4.36^{\circ}$ & $9.36 \pm 4.47^{\circ}$ & 0.87 \\
\hline Pelvic rotation peak angle & $6.77 \pm 2.46^{\circ}$ & $6.78 \pm 3.58^{\circ}$ & 0.99 \\
\hline Lumbar extension peak angle & $10.48 \pm 6.15^{\circ}$ & $10.75 \pm 5.67^{\circ}$ & 0.54 \\
\hline Lumbar rotation peak angle & $7.15 \pm 2.23^{\circ}$ & $7.12 \pm 2.48^{\circ}$ & 0.92 \\
\hline Step length $(\mathrm{cm})$ & $70.0 \pm 5.7$ & $71.8 \pm 3.7$ & 0.21 \\
\hline
\end{tabular}


Table 4: Between-group comparison for changes in kinematic parameters.

\begin{tabular}{|l|l|l|l|l|}
\hline & Stretching group $(\mathbf{n}=10)$ & Control group $(\mathbf{n}=10)$ & p value & Effect size (95\% Cl) \\
\hline Hip extension peak angle & $0.27 \pm 1.24^{\circ}$ & $0.05 \pm 0.75^{\circ}$ & 0.65 & $0.21(-0.24$ to 0.66$)$ \\
\hline Pelvic anterior tilt peak angle & $-0.06 \pm 1.19^{\circ}$ & $-0.03 \pm 0.46^{\circ}$ & 0.94 & $0.03(-0.36$ to 0.43$)$ \\
\hline Pelvic rotation peak angle & $0.25 \pm 1.31^{\circ}$ & $0.01 \pm 1.82^{\circ}$ & 0.75 & $-0.15(-0.84$ to 0.55$)$ \\
\hline Lumbar extension peak angle & $-0.20 \pm 1.77^{\circ}$ & $0.27 \pm 1.27^{\circ}$ & 0.53 & $0.29(-0.38$ to 0.97$)$ \\
\hline Lumbar rotation peak angle & $0.78 \pm 0.88^{\circ}$ & $-0.03 \pm 0.90^{\circ}$ & 0.07 & $-0.87(-1.26 \text { to }-0.48)^{*}$ \\
\hline Step length $(\mathrm{cm})$ & $1.0 \pm 2.9$ & $1.8 \pm 4.0$ & 0.62 & $-0.22(-1.75$ to 1.31$)$ \\
\hline
\end{tabular}

${ }^{*} 95 \% \mathrm{Cl}$ did not include the value of zero.

values between the two groups, which guaranteed the baseline comparability. As a result of within-group comparisons utilising paired t-tests, both two groups did not show any significant change in lumbopelvic-hip kinematics after stretching or control interventions (see Table 3). Between-group comparisons showed no significant difference in any outcome measures in terms of $p$ values (see Table 4). However, our additional analysis revealed that $95 \% \mathrm{Cl}$ of between-group effect size in peak lumbar rotation angle did not included zero (see Table 4). This implied a significantly greater increase in peak lumbar rotation angle in the stretching group compared to the control group.

\section{Discussion}

The aim of this study was to investigate acute effects of active stretching for iliopsoas muscles on lumbopelvic-hip kinematics of subjects with non-specific low back pain and reduced hip extension. The findings of this research suggest that a single set of active stretching might not be effective to acutely improve hip extension of patients with non-specific low back pain. Although there was no statistically significant difference $(p=$ 0.07), this study also implied that active stretching for iliopsoas muscles can lead to increased lumbar rotation during gait.

To the best of the authors' knowledge, this was the first study which investigated the acute effects of active stretching for iliopsoas muscles on lumbopelvic-hip kinematics of patients with non-specific low back pain and reduced hip extension. Although one randomised controlled trial suggested that ten weeks of stretching programs can result in increased hip extension in gait or functional movements of asymptomatic subjects [11], this study failed to demonstrate short-term effectiveness of the same stretching technique for symptomatic subjects. As this study did not adopt long-term follow-ups, this discrepancy in the results might have derived from the insufficient length of the intervention. Another possibility is that the dosage of the stretching intervention was not sufficient to elicit detectable changes. The study by Kerrigan, et al. used four sets of 30-second stretches, whilst the stretching maneuver in this study was held for $20 \mathrm{~s}$. Therefore, further study with more vigorous stretching techniques is required. Although exploring the effects of passive stretching techniques might be also helpful [21], the reliability of measuring methods for kinematic parameters can be compromised, for it would be difficult to perform passive interventions without affecting the locations of markers.

Additional analysis using effect sizes suggested that active stretching for iliopsoas muscles can lead to increased lumbar rotation during gait. Considering that there was no significant change in pelvic rotation in stretching group, this change might derive from changes in kinematics of upper trunk rotation. However, it is not entirely clear with regard to the mechanical mechanism why this particular stretching for lower limbs can increase upper trunk rotation during gait (refer to Figure 3 ). Since there was a discrepancy between the results from $p$ values and those from effect sizes (see Table 4), these data must be interpreted with caution.

\section{Limitations of the study}

The present study may be limited by the small sample size $(n=10)$. A potentially insufficient sample size might have compromised the statistical precision, leading to inaccurate results [22]. However, the findings of this study can be used to estimate necessary sample size to achieve sufficient statistical power in further research [23]. Since this study did not use long-term interventions and follow-ups, long-term effects of stretching interventions are still unclear from this study. Another limitation is the lack of blinding for participants and assessors. This study is also limited by the inconsistent results regarding lumbar rotation peak angle, where $p$ value indicated statistical insignificance $(p=0.07)$, whilst its effect size implied statistical significance. These contradictory findings should be interpreted carefully. Another limitation lies in potentially varied gait speed, which was not controlled in testing sessions. Lastly, this study may be limited by the fact that other hip flexors, such as rectus femoris and tensor fascia lata were not stretched. Potential stiffness of these muscles among participants might have confounded the results.

\section{Clinical implications}

Based on the findings in our study, a single set of active stretching for iliopsoas muscles, adopted in this study, might not be effective to increase hip extension of patients with non-specific low back pain. Hence, this type of stretching might not be effective to reduce mechanical stress to lumbar spine during gait. Further research is required to justify the long-term use of this 
stretching method to improve hip extension and control lumbopelvic movements of patients with non-specific low back pain, which is exacerbated by extension-related activities.

\section{Conclusions}

This study implies that a single set of active static stretching might not immediately improve hip extension or alter lumbopelvic movements of patients with non-specific low back pain during gait. Further study with better methodological rigour is required to test the inconclusive findings in this study.

\section{Conflict of Interests}

The authors declare no conflict of interests.

\section{Funding}

The present study was supported by Tokyo University of Technology.

\section{Acknowledgement}

We would like to thank Tokyo University of Technology for providing funding for this research project. We would also like to thank Mr Daiki Shimotori and Mr Kazuya Matsushita for helpful instructions regarding 3-D motion analysis.

\section{References}

1. Buchbinder R, Blyth FM, March LM, Brooks $P$, Woolf $A D$, et al. (2013) Placing the global burden of low back pain in context. Best Pract Res Clin Rheumatol 27: 575-589.

2. Vos $T$, Flaxman $A D$, Naghavi M, Lozano R, Michaud $C$, et al. (2012) Years lived with disability (YLDs) for 1160 sequelae of 289 diseases and injuries 1990-2010: a systematic analysis for the Global Burden of Disease Study 2010. Lancet 380: 2163-2196.

3. Airaksinen $\mathrm{O}$, Brox $\mathrm{JI}$, Cedraschi $\mathrm{C}$, Hildebrandt $\mathrm{J}$, Klaber-Moffett J, et al. (2006) Chapter 4. European guidelines for the management of chronic nonspecific low back pain. Eur Spine J 15: S192-S300.

4. Page P, Frank C, Lardner R (2010) Assessment and treatment of muscle imbalance: the Janda approach: Human kinetics.

5. Peterson-Kendall F, Kendall-McCreary E, Geise-Provance $P$ (2005) Muscles testing and function with posture and pain: Baltimore: Lippincott Williams \& Wilkins.

6. Sahrmann S (2002) Diagnosis and treatment of movement impairment syndromes: Elsevier Health Sciences.

7. O'sullivan $P(2000)$ Masterclass. Lumbar segmental 'instability': clinical presentation and specific stabilizing exercise management. Manual Therapy 5: 2-12.

8. Godges JJ, Macrae H, Longdon C, Tinberg C, Macrae PG (1989) The effects of two stretching procedures on hip range of motion and gait economy. J Orthop Sports Phys Ther 10: 350-357.

9. Moreside JM, McGill SM (2013) Improvements in hip flexibility do not transfer to mobility in functional movement patterns. J Strength Cond Res 27: 2635-2643.

10. Standaert CJ, Herring SA (2007) Expert opinion and con- troversies in sports and musculoskeletal medicine: the diagnosis and treatment of spondylolysis in adolescent athletes. Arch Phys Med Rehabil 88: 537-540.

11. Kerrigan DC, Xenopoulos-Oddsson A, Sullivan MJ, Lelas JJ, Riley PO (2003) Effect of a hip flexor-stretching program on gait in the elderly. Arch Phys Med Rehabil 84: 1-6.

12. Merlin T, Weston A, Tooher R (2009) Extending an evidence hierarchy to include topics other than treatment: revising the Australian 'levels of evidence'. BMC Med Res Methodol 9: 34.

13. Clapis PA, Davis SM, Davis RO (2008) Reliability of inclinometer and goniometric measurements of hip extension flexibility using the modified Thomas test. Physiother Theory Pract 24: 135-141.

14. Barker S, Craik R, Freedman W, Herrmann N, Hillstrom H (2006) Accuracy, reliability, and validity of a spatiotemporal gait analysis system. Med Eng Phys 28: 460-467.

15. Myers KA, Long JT, Klein JP, Wertsch JJ, Janisse D, et al. (2006) Biomechanical implications of the negative heel rocker sole shoe: gait kinematics and kinetics. Gait Posture 24: 323-330.

16. Maher CG, Sherrington C, Herbert RD, Moseley AM, Elkins M (2003) Reliability of the PEDro scale for rating quality of randomized controlled trials. Phys Ther 83: 713-721.

17. Norman G (2010) Likert scales, levels of measurement and the "laws" of statistics. Adv Health Sci Educ Theory Pract 15: 625-632.

18. Hedges LV (1981) Distribution theory for Glass's estimator of effect size and related estimators. Journal of Educational Statistics 6: 107-128.

19. Nakagawa S, Cuthill IC (2007) Effect size, confidence interval and statistical significance: a practical guide for biologists. Biol Rev Camb Philos Soc 82: 591-605.

20. Cohen J (1992) A power primer. Psychol Bull 112: 155-159.

21. Minshull C, Eston R, Bailey A, Rees D, Gleeson N (2014) The differential effects of PNF versus passive stretch conditioning on neuromuscular performance. Eur J Sport Sci 14: 233-241.

22. Button KS, loannidis JP, Mokrysz C, Nosek BA, Flint J, et al. (2013) Power failure: why small sample size undermines the reliability of neuroscience. Nat Rev Neurosci 14: 365376.

23. Batterham AM, Atkinson G (2005) How big does my sample need to be? A primer on the murky world of sample size estimation. Physical Therapy in Sport 6: 153-163. 